\title{
Evaluation of New High-Producing Hybrids of Cocoa Trees in Marginal Crop Areas in Côte d'Ivoire
}

\author{
Stanislas T. Kouame ${ }^{1,2 *}$, Mathias G. Tahi' ${ }^{2}$, Aïdara Sekou ${ }^{2}$, Christian Cilas ${ }^{3}$, Selastique D. Akaffou ${ }^{1}$ \\ ${ }^{1}$ Laboratory for Agricultural Production Improvement, Agroforestry Research and Training Unit, Jean Lorougnon Guede \\ University, Daloa, Côte d'Ivoire \\ ${ }^{2}$ Laboratory of Genetics, Divo Research Station, National Center for Agronomic Research (CNRA), Divo, Ivory Coast \\ ${ }^{3} \mathrm{CIRAD}$, Center for International Cooperation in Agricultural Research for Development, Montpellier, France \\ Email: *stane.kouame@gmail.com
}

How to cite this paper: Kouame, S.T., Tahi, M.G., Sekou, A., Cilas, C. and Akaffou, S.D. (2021) Evaluation of New High-Producing Hybrids of Cocoa Trees in Marginal Crop Areas in Côte d'Ivoire. Agricultural Sciences, 12, 1508-1526. https://doi.org/10.4236/as.2021.1212096

Received: August 30, 2021

Accepted: December 20, 2021

Published: December 23, 2021

Copyright (c) 2021 by author(s) and Scientific Research Publishing Inc. This work is licensed under the Creative Commons Attribution International License (CC BY 4.0).

http://creativecommons.org/licenses/by/4.0/

\begin{abstract}
A study was carried out on 31 new hybrids in rain-deficient areas of Côte d'Ivoire. These areas were the regions of Djekanou, Toumodi and Yamoussoukro. The Divo region (favourable zone) was used as a control. The aim of this study was to determine the behaviour of these hybrids under drought conditions. The experimental setup was a Fischer block for each region. The treatments were the 31 families, with 3 replications and when the four regions were taken together, the study was a two-factor (region and family) trial. Drought caused increased mortality and specific leaf mass and reduced growth and development of progeny in rain-deficient areas. Five (5) progenies were found to be jointly successful in Divo, Djekanou and Yamoussoukro (F30, F15, F19, F31, and F33). On the other hand, 3 other families (F16, F36 and F29) also performing well in Divo were found in Toumodi.
\end{abstract}

\section{Keywords}

Drought, Resistant Hybrids, Theombro cacao, Côte d'Ivoire

\section{Introduction}

In recent years, with the onset of drought due to climate change, cocoa cultivation has been severely hampered in some areas in Côte d'Ivoire. The drought caused the deterioration of cocoa growing conditions with high mortality and a drop in production [1].

In this context of drought, the efforts of producers were in vain due to the lack of suitable plant material [2] [3]. When the minimum conditions for cocoa cul- 
tivation are met again in these marginal areas, then the return of this cultivation will be a great opportunity to reduce the pressure on the forest. Indeed, Ivorian forest cover has deteriorated considerably over the past 25 years with a conservation rate dropping from 16 million to 3.5 million hectares or $21.88 \%$ [4].

The cocoa program of the National Agronomic Research Center (CNRA) of Côte d'Ivoire, has produced high-performance varieties [5]. While these varieties tolerate certain biotic hazards such as diseases [6], their behavior in the face of drought linked to climate change is not understood. The purpose of this study is to assess the drought behavior of 31 new hybrids. Specifically, this involves determining mortality and evaluating the development and growth of hybrids.

\section{Material and Methods}

\subsection{Plant Material}

The plant material consists of 31 progenies obtained by controlled fertilization (Table 1). Thirty-six (36) plants were evaluated per progeny. A total of 4092 genotypes spread over 4 plots in four experimental sites with different rainfall patterns were monitored. These plants were installed on trial in 2018. They were 2 years old after planting at the time of the assessments.

Table 1. Descendants resulting from crosses between the parents.

\begin{tabular}{cccc}
\hline & Manual crossing & & Progenies codes \\
\cline { 1 - 3 } Females & & Males & F1 \\
\hline UPA402 & $\mathrm{X}$ & UF676 & F2 \\
UPA409 & $\mathrm{X}$ & IFC1 & F3 \\
UPA608 & $\mathrm{X}$ & IFC412 & F5 \\
UPA603 & $\mathrm{X}$ & UF667 & F7 \\
T85/799 & $\mathrm{X}$ & IFC15 & F8 \\
SCA6 & $\mathrm{X}$ & ICS1 & F9 \\
PA150 & $\mathrm{X}$ & IFC5 & F10 \\
T79/501 & $\mathrm{X}$ & IFC5 & F11 \\
IFC720 & $\mathrm{X}$ & ICS46 & F12 \\
IMC67 & $\mathrm{X}$ & IFC1 & F13 \\
MOQ413 & $\mathrm{X}$ & SCA6 & F14 \\
POR & $\mathrm{X}$ & T60/887 & F15 \\
PA150 & $\mathrm{X}$ & POR & F16 \\
IFC303 & $\mathrm{X}$ & IFC1 & F17 \\
SCA6 & $\mathrm{X}$ & LAF1 & F19 \\
T60/887 & $\mathrm{X}$ & IMC67 & F20 \\
PA150 & $\mathrm{X}$ & T60/887 & F21 \\
P7 & $\mathrm{X}$ & GU175A & \\
\hline
\end{tabular}




\section{Continued}

\begin{tabular}{cccc}
\hline P7 & X & GU284 A & F22 \\
IFC303 & X & GU284 A & F24 \\
P7 & X & IMC67 & F25 \\
P7 & X & SCA6 & F26 \\
T60/887 & X & IFC5 & F27 \\
NA32 & X & IFC1 & F28 \\
PA150 & X & T79/501 & F29 \\
T60/887 & X & POR & F30 \\
UPA413 & X & SCA6 & F31 \\
P7 & X & T60/887 & F32 \\
P7 & X & PA150 & F33 \\
IMC67 & $X$ & T60/887 & F35 \\
POR & X & PA150 & F36
\end{tabular}

\subsection{Methods}

\subsubsection{Study Sites}

The study was undertaken in four sites: Divo, Djekanou, Toumodi, and Yamoussoukro (Figure 1). The first site belongs to the favorable zone of cocoa culture in Côte d'Ivoire while the three later were found in unfavorable (marginal zone). Choice of plots for the study in the marginal zone was made by following the classification scale of favorable zones to cocoa cultivation. Four areas were chosen. These are Djekanou, Toumodi, Yamoussoukro (unfavorable area) and Divo (favorable area to cocoa cultivation and normal rainfall) which serves as a witness in our experiment. Table 2 presents the climatic characteristics (rainfall and temperature) of these sectors.

\subsubsection{Experimentation}

The experimental design was a Fischer block for each region. The treatments were the 31 families, with 3 replicates of 12 trees each, for a total of 36 trees per family, for Divo, Djekanou and Toumodi and 3 replicates of 8 trees for Yamoussoukro. The standard density of 1333 trees per hectare $(3 \mathrm{~m}$ between the rows and $2.5 \mathrm{~m}$ between the plants) was respected. When the four regions were taken together, the study was a two-factor (region and family) trial. A total of 4092 genotypes were evaluated.

\subsubsection{Crop Management}

The transfer of the seedlings to the different study sites was carried out after six months of residence of the seedlings in the nursery. Planting concerned vigorous plants. Plot maintenance consisted of manual weeding and the use of insecticide products (Califan super solicao and pyrical) as soon as symptoms of parasitism appeared, and of foliar fertiliser (califere) at a rate of $100 \mathrm{ml}$ of insecticide in a 10-litre water sprayer. 


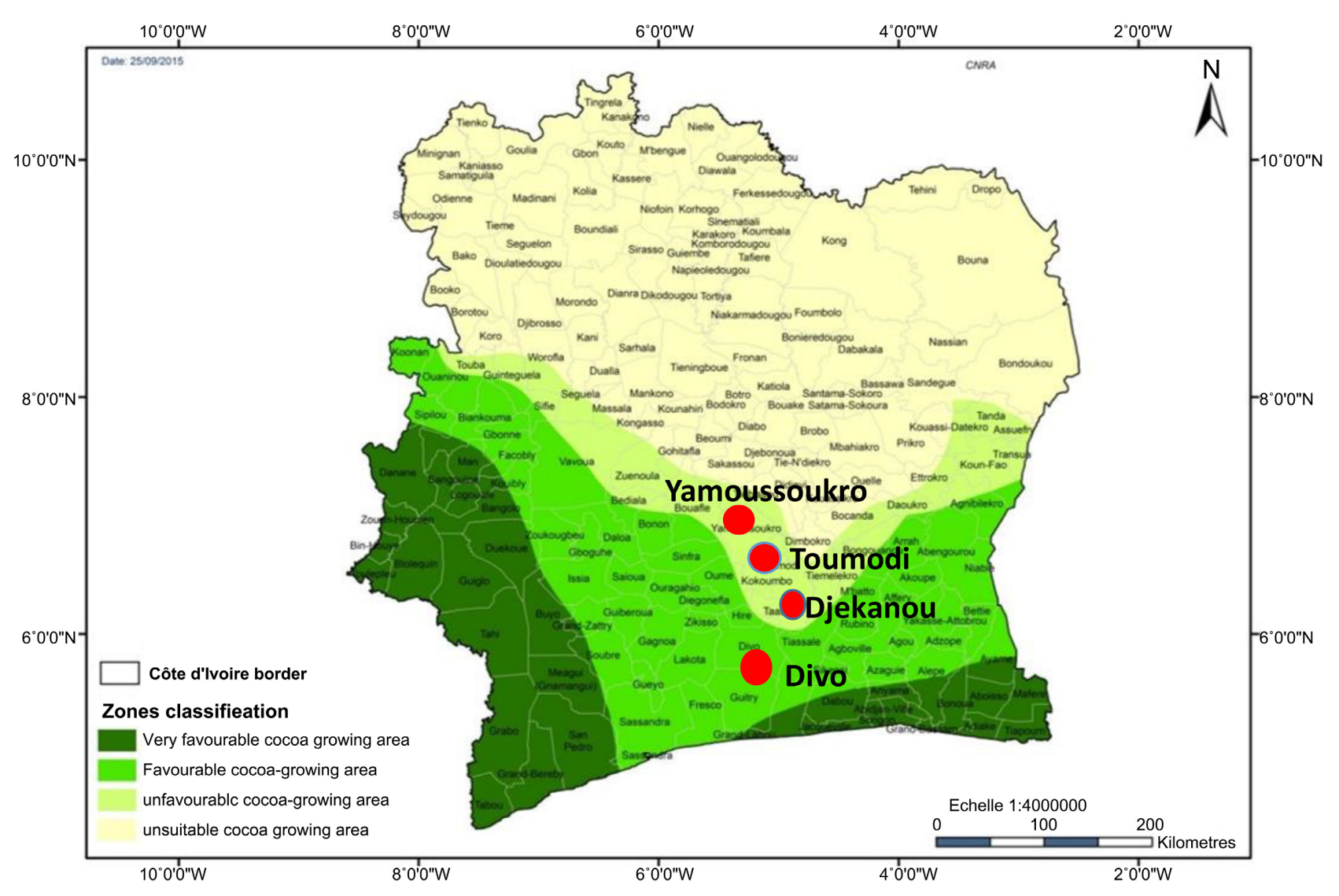

Figure 1. Study area.

Table 2. Rainfall and temperature of regions hosting the study sites.

\begin{tabular}{ccccccc}
\hline & \multicolumn{3}{c}{ Rainfall $(\mathrm{mm})$} & \multicolumn{3}{c}{ Temperatures $\left({ }^{\circ} \mathrm{C}\right)$} \\
\hline & Mini & Maxi & Mean & Mini & Maxi & Mean \\
\hline Divo & 24 & 249 & 1469 & 24.5 & 27.6 & 26.2 \\
Djekanou & 12 & 182 & 1092 & 25.2 & 28.3 & 26.6 \\
Toumodi & 12 & 180 & 1091 & 25.5 & 28.4 & 26.5 \\
Yamoussoukro & 12 & 170 & 1118 & 25.5 & 28.8 & 26.9 \\
\hline
\end{tabular}

\subsubsection{Parameters Assessed}

Three types of parameters were evaluated: mortality rate, growth and development, and physiological parameters.

\section{$\checkmark$ Mortality Rate}

The mortality rate was calculated 24 months after field planting. It is expressed as a percentage and corresponds to the ratio between the number of dead plants and the number of plants planted.

\section{$\checkmark$ Growth and Development}

This is the collar diameter, the height of the plants, the crown rate and the leaf dimensions.

The collar diameter was measured at $10 \mathrm{~cm}$ from the ground with a caliper. 
Plant height was measured from the ground to the apex of the trunk with a tape measure. The crown rate, expressed as a percentage, is the ratio of the number of crowned plants to the number of plants planted. The crown is the part of the cocoa tree that consists of a structured set of master branches or secondary shoots located at the top of the trunk. The height of the crown was measured with a tape measure; it is the length between the crown and the collar. The number of functional leaves per plant was counted. These are the green leaves, capable of photosynthesis. The dimensions, length and width of the leaves (three leaves per plant) were measured with a ruler and the leaf area was calculated according to the formula of [7]:

$$
S\left(\mathrm{~cm}^{2}\right)=\frac{\pi a b}{4}
$$

where $a=A / 2$ and $b=B / 2 ; A=$ length, $B=$ width. The total leaf area of the leaves was calculated by adding the elemental areas.

Physiological parameters (water content and leaf density)

The water content of the leaves was calculated by the following formula:

$$
T E(\mathrm{mg} / \mathrm{gMs})=\frac{(M F-M s)}{M s}
$$

with $T E=$ water content; $M f=$ fresh mass and $M s=$ dry mass.

The specific leaf mass (SLM) was determined by the formula of [8]:

$$
S L M\left(\mathrm{mg} / \mathrm{cm}^{2}\right)=\frac{M F}{S F}
$$

with $M F=$ fresh mass and $S F=$ leaf area.

\subsubsection{Statistical Analysis of the Data}

The comparison of the hybrid families was done by analysis of variance, with a threshold $\mathrm{p}=0.05$. Pearson correlation was used to estimate the relationships between the agro-morphological and physiological variables. Principal component analysis (PCA) and hierarchical ascending classification (HAC) were used to reveal the strongest correlations between the variables and to assess the most discriminating ones for efficient selection of cocoa varieties at a young age, both overall and within the regions. These analyses were performed with SAS 9.4 (SAS Institute, 2018) and XLSTAT version 2014.

\section{Results}

\subsection{Influence of Areas on the Behaviour of Young Cocoa Trees}

The regions on all the families showed significant differences between them for all the parameters evaluated. The region * family interactions were significant for the parameters diameter at the collar, plant height, number of living leaves with a probability $<0.0001$ and mortality rate with a probability of 0.01047 . On the other hand, region * family interactions were not significant for the parameters crown rate (0.4847), crown height (0.8265), leaf area (0.9683), water content 
(0.9961) and leaf specific gravity (0.8993) (Table 3 ). The mortality rate of young cocoa plants, twenty-four months after their establishment in the field, varied significantly according to the agro-climatic zones. It averaged $12.7 \%$ in Divo, which recorded the lowest average for all 31 families, compared to $68.01 \%$ for the Yamoussoukro zone, which showed the highest rate. Djekanou and Toumodi had intermediate averages of $21.9 \%$ and $49.7 \%$ respectively. The largest collar diameters were obtained by Divo with $1.64 \mathrm{~cm}$ and Toumodi with $1.6 \mathrm{~cm}$. On the other hand, the smallest collar diameters were presented by the regions of Djekanou with $1.36 \mathrm{~cm}$ and Yamoussoukro with $1.28 \mathrm{~cm}$. For plant height, Divo region had the highest value with $103.05 \mathrm{~cm}$, and Yamoussoukro region the lowest with $70.26 \mathrm{~cm}$. The number of living leaves was highest in Divo with 28.14 leaves, Djekanou with 23.86 leaves and Yamoussoukro with 22.77 leaves. On the other hand, the Toumodi region, with 9.58 live leaves, had the lowest number. For the crown rate, the regions of Djekanou with $14.39 \%$, Divo with $12.44 \%$ and Toumodi with $10.48 \%$ were the best. The Yamoussoukro region with $3.80 \%$ had the lowest crowning rate. In terms of crown height, Divo with $70.87 \mathrm{~cm}$ and Djekanou with $67.05 \mathrm{~cm}$ were the best. The Yamoussoukro region with $13.36 \mathrm{~cm}$ had the lowest crown height. For leaf area, Divo region had the largest with $108.96 \mathrm{~cm}^{2}$ and Yamoussoukro $\left(51.32 \mathrm{~cm}^{2}\right)$ and Toumodi $(43.15$ $\mathrm{cm}^{2}$ ) regions had the smallest areas. The highest water content was presented by the Divo region with $3951.5 \mathrm{mg} / \mathrm{g}$ and the lowest by the Yamoussoukro (1129.8 $\mathrm{mg} / \mathrm{g}$ ) and Toumodi $(746.6 \mathrm{mg} / \mathrm{g})$ regions. The highest specific leaf mass was obtained by the Yamoussoukro region with $7713 \mathrm{mg} / \mathrm{cm}^{2}$ and the lowest by the Divo region with $656.6 \mathrm{mg} / \mathrm{cm}^{2}$ (Table 3). The regions of Djekanou and Toumodi presented intermediate averages of $1600 \mathrm{mg} / \mathrm{cm}^{2}$ and $1380.2 \mathrm{mg} / \mathrm{cm}^{2}$ respectively.

Table 3. Comparison of experimental areas according to growth and development parameters of cocoa plants.

\begin{tabular}{|c|c|c|c|c|c|c|c|c|c|}
\hline \multirow[b]{2}{*}{ Area } & \multirow[b]{2}{*}{$\begin{array}{c}\text { Rates of } \\
\text { mortality } \\
\%\end{array}$} & \multicolumn{6}{|c|}{ Growth and development parameters } & \multicolumn{2}{|c|}{ Physiological parameters } \\
\hline & & $\begin{array}{c}\text { Collar } \\
\text { Diameter } \\
(\mathrm{cm})\end{array}$ & $\begin{array}{l}\text { height } \\
\text { of plants } \\
(\mathrm{cm})\end{array}$ & $\begin{array}{c}\text { Rates } \\
\text { of crown } \\
(\%)\end{array}$ & $\begin{array}{l}\text { height } \\
\text { of crown } \\
(\mathrm{cm})\end{array}$ & $\begin{array}{c}\text { number } \\
\text { of living } \\
\text { leaves }\end{array}$ & $\begin{array}{c}\text { Total } \\
\text { leaf area } \\
\left(\mathrm{cm}^{2}\right)\end{array}$ & $\begin{array}{c}\text { Water } \\
\text { Content } \\
(\mathrm{mg} / \mathrm{g})\end{array}$ & $\begin{array}{l}\text { Specific } \\
\text { leaf mass } \\
\left(\mathrm{mg} / \mathrm{cm}^{2}\right)\end{array}$ \\
\hline Divo & $12.7 \pm 10^{\mathrm{a}}$ & $1.64 \pm 0.25^{\mathrm{a}}$ & $103.05 \pm 10.66^{\mathrm{a}}$ & $12.44 \pm 6.4^{\mathrm{a}}$ & $70.87 \pm 33^{\mathrm{a}}$ & $28.14 \pm 10.47^{\mathrm{a}}$ & ${ }^{\mathrm{a}} 108.96 \pm 35.53^{\mathrm{a}}$ & $\mathrm{a} 3951.5 \pm 1195.95$ & $656.6 \pm 11.54^{\mathrm{c}}$ \\
\hline Djekanou & $21.9 \pm 9^{b}$ & $1.36 \pm 0.16^{\mathrm{b}}$ & $87.89 \pm 11.94^{\mathrm{c}} 1$ & $14.39 \pm 8.92^{\mathrm{a}} \mathrm{C}$ & $67.05 \pm 32.35^{\mathrm{a}}$ & $23.86 \pm 8.43^{\mathrm{b}}$ & $67.52 \pm 19.02^{\mathrm{b}}$ & $2306.7 \pm 490.05^{\mathrm{b}}$ & b $1600.8 \pm 24.34^{b}$ \\
\hline Toumodi & $49.7 \pm 17^{\mathrm{c}}$ & $1.60 \pm 0.25^{\mathrm{a}}$ & $95.19 \pm 13.08^{\mathrm{b}} 1$ & $10.48 \pm 7.65^{\mathrm{a}}$ & $47.73 \pm 23.32^{b}$ & $9.58 \pm 4.54^{\mathrm{d}}$ & $43.15 \pm 27.72^{c}$ & $746.6 \pm 348.17^{c}$ & $1380.2 \pm 23.37^{\mathrm{b}}$ \\
\hline Yamoussoukro & $68.01 \pm 14.6^{\mathrm{d}}$ & d $1.28 \pm 0.29^{c}$ & $70.26 \pm 21.35^{c}$ & $3.80 \pm 6.03^{\mathrm{b}}$ & $13.36 \pm 20.42^{\mathrm{c}}$ & ${ }^{c} 22.77 \pm 17.58^{c}$ & ${ }^{c} 51.32 \pm 34.95^{\mathrm{cb}}$ & $1129.8 \pm 674.77^{c}$ & $7713 \pm 75.47^{\mathrm{a}}$ \\
\hline Mean & 38 & 1.51 & 93.66 & 11.06 & 48.74 & 22.36 & 76.57 & 2497.59 & 1710.8 \\
\hline $\mathrm{CV}(\%)$ & 49.52 & 36.56 & 34.14 & 13.93 & 81.56 & 43.15 & 133.91 & 154.24 & 103.25 \\
\hline $\mathrm{p} \leq 0.05$ & $<0.0001$ & $<0.0001$ & $<0.0001$ & 0.0045 & $<0.0001$ & $<0.0001$ & $<0.0001$ & $<0.0001$ & $<0.0001$ \\
\hline Region * family & 0.01047 & $<0.0001$ & $<0.0001$ & 0.4847 & 0.8265 & $<0.0001$ & 0.9683 & 0.9961 & 0.8993 \\
\hline
\end{tabular}

In each column, the means followed by the same letter are not statistically different at the $5 \%$ level. 


\subsection{Growth and Development of Cocoa Trees in All Regions with Non-Significant Region* Family Interactions}

The parameters with non-significant region ${ }^{*}$ family interactions were crown rate, crown height, leaf area, water content and leaf specific mass. For these parameters, the families across all regions showed significant differences between them. Thus, the rankings of the families across all regions are the same for each region. For the crown rate, families F25 with $29.84 \%$, F29 with $19.32 \%$ and F19 with $17.32 \%$ had the best crown rates. On the other hand, the lowest rates were obtained by families F17 (5.93\%), F1 (5.76\%) and F2 (5.14\%). For crown height, families F35 with $76.59 \mathrm{~cm}$ and F22 with $70.03 \mathrm{~cm}$ had the best crown heights. On the other hand, the lowest height was obtained by family F24 with $11.45 \mathrm{~cm}$. In terms of leaf area, families F19 with $115.12 \mathrm{~cm}^{2}$ and F36 with $108.04 \mathrm{~cm}^{2}$ had the largest areas. On the other hand, the smallest area was obtained by family F10 with $38.94 \mathrm{~cm}^{2}$. Families F1 with $3468.1 \mathrm{mg} / \mathrm{g}$ and F19 with $3428.5 \mathrm{mg} / \mathrm{g}$ had the highest water content and families F10 $(1486.7 \mathrm{mg} / \mathrm{g})$ and F28 $(1582.4 \mathrm{mg} / \mathrm{g})$ the lowest. Families F36 $\left(8088 \mathrm{mg} / \mathrm{cm}^{2}\right)$, F19 $\left(8758 \mathrm{mg} / \mathrm{cm}^{2}\right)$, F32 $\left(8527 \mathrm{mg} / \mathrm{cm}^{2}\right)$, F16 $\left(8329 \mathrm{mg} / \mathrm{cm}^{2}\right), F 15\left(8134 \mathrm{mg} / \mathrm{cm}^{2}\right)$ had the highest specific leaf masses. On the other hand, families F13 $\left(8.74 \mathrm{mg} / \mathrm{cm}^{2}\right), \mathrm{F} 1\left(10.84 \mathrm{mg} / \mathrm{cm}^{2}\right)$ and F12 $(12.37$ $\mathrm{mg} / \mathrm{cm}^{2}$ ) had the lowest masses (Table 4).

Table 4. Comparison of families across regions according to parameters with non-significant region * family interaction.

\begin{tabular}{cccccc}
\hline Family & $\begin{array}{c}\text { Crown } \\
\text { rate }(\%)\end{array}$ & $\begin{array}{c}\text { Crown } \\
\text { height }(\mathbf{c m})\end{array}$ & $\begin{array}{c}\text { Total leaf } \\
\text { area }\left(\mathrm{cm}^{2}\right)\end{array}$ & $\begin{array}{c}\text { Water Content } \\
(\mathbf{m g} / \mathrm{g})\end{array}$ & $\begin{array}{c}\text { Specific leaf } \\
\text { mass }\left(\mathbf{m g} / \mathrm{cm}^{2}\right)\end{array}$ \\
\hline F1 & $5.76 \mathrm{c}$ & $26.9 \mathrm{bc}$ & $89.92 \mathrm{ba}$ & $3468.1 \mathrm{a}$ & $10.84 \mathrm{c}$ \\
F10 & $11.88 \mathrm{bc}$ & $35.35 \mathrm{bac}$ & $38.94 \mathrm{c}$ & $1486.7 \mathrm{c}$ & $19.75 \mathrm{bac}$ \\
F11 & $10.2 \mathrm{bc}$ & $53.65 \mathrm{ba}$ & $68.34 \mathrm{bac}$ & $2820.4 \mathrm{ba}$ & $16.63 \mathrm{bac}$ \\
F12 & $9.78 \mathrm{bc}$ & $53.82 \mathrm{ba}$ & $59.15 \mathrm{bac}$ & $1894.6 \mathrm{bc}$ & $12.37 \mathrm{c}$ \\
F13 & $12.69 \mathrm{bac}$ & $66.8 \mathrm{ba}$ & $76.45 \mathrm{ba}$ & $2837.8 \mathrm{ba}$ & $8.74 \mathrm{c}$ \\
F14 & $12.14 \mathrm{bac}$ & $31.32 \mathrm{bac}$ & $90.14 \mathrm{ba}$ & $2730.3 \mathrm{bac}$ & $20.46 \mathrm{bac}$ \\
F15 & $12.82 \mathrm{bac}$ & $67.01 \mathrm{ba}$ & $87.01 \mathrm{ba}$ & $2908.7 \mathrm{ba}$ & $8134 \mathrm{a}$ \\
F16 & $12.86 \mathrm{bac}$ & $64.34 \mathrm{ba}$ & $97.49 \mathrm{ba}$ & $2777.3 \mathrm{bac}$ & $8329 \mathrm{a}$ \\
F17 & $5.93 \mathrm{c}$ & $35.21 \mathrm{bac}$ & $75.6 \mathrm{ba}$ & $2952.5 \mathrm{ba}$ & $7951 \mathrm{ba}$ \\
F19 & $17.32 \mathrm{ba}$ & $62.73 \mathrm{ba}$ & $115.12 \mathrm{a}$ & $3428.5 \mathrm{a}$ & $8758 \mathrm{a}$ \\
F2 & $5.14 \mathrm{c}$ & $26.92 \mathrm{bc}$ & $67.12 \mathrm{bac}$ & $2633.1 \mathrm{bac}$ & $6934 \mathrm{bac}$ \\
F20 & $11.62 \mathrm{bc}$ & $65.58 \mathrm{ba}$ & $80.59 \mathrm{ba}$ & $2541.3 \mathrm{bac}$ & $7585 \mathrm{ba}$ \\
F21 & $7.8 \mathrm{bc}$ & $48.17 \mathrm{bac}$ & $74.88 \mathrm{ba}$ & $2188 \mathrm{bac}$ & $6185 \mathrm{bac}$ \\
F22 & $8.48 \mathrm{bc}$ & $70.03 \mathrm{a}$ & $69.14 \mathrm{bac}$ & $2597.1 \mathrm{bac}$ & $6690 \mathrm{bac}$ \\
F24 & $14.6 \mathrm{bac}$ & $11.45 \mathrm{c}$ & $74.03 \mathrm{ba}$ & $2267.5 \mathrm{bac}$ & $6400 \mathrm{bac}$ \\
F25 & $29.84 \mathrm{a}$ & $65.03 \mathrm{ba}$ & $70.06 \mathrm{ba}$ & $1971.6 \mathrm{bc}$ & $5405 \mathrm{bc}$ \\
F26 & $12 \mathrm{bac}$ & $57.21 \mathrm{ba}$ & $79.76 \mathrm{bac}$ & $1911.6 \mathrm{bc}$ & $6159 \mathrm{bac}$ \\
\hline
\end{tabular}




\begin{tabular}{cccccc} 
Continued & \multicolumn{5}{l}{} \\
\hline F27 & $10.4 \mathrm{bc}$ & $33.08 \mathrm{bac}$ & $60.04 \mathrm{bac}$ & $1934.7 \mathrm{bc}$ & $5818 \mathrm{bc}$ \\
F28 & $6.64 \mathrm{bc}$ & $61.99 \mathrm{ba}$ & $39.75 \mathrm{bc}$ & $1582.4 \mathrm{c}$ & $4238 \mathrm{c}$ \\
F29 & $19.32 \mathrm{ba}$ & $45.54 \mathrm{bac}$ & $71.06 \mathrm{ba}$ & $2341.9 \mathrm{bac}$ & $6575 \mathrm{bac}$ \\
F3 & $14.72 \mathrm{bac}$ & $43.14 \mathrm{bac}$ & $77.63 \mathrm{ba}$ & $2245.2 \mathrm{bac}$ & $6598 \mathrm{bac}$ \\
F30 & $10.47 \mathrm{bc}$ & $66.95 \mathrm{ba}$ & $97.51 \mathrm{ba}$ & $2726.4 \mathrm{bac}$ & $7880 \mathrm{ba}$ \\
F31 & $12.41 \mathrm{bac}$ & $45.38 \mathrm{bac}$ & $76.63 \mathrm{ba}$ & $2688.2 \mathrm{bac}$ & $7236 \mathrm{ba}$ \\
F32 & $7.76 \mathrm{bc}$ & $35.29 \mathrm{bac}$ & $88.29 \mathrm{ba}$ & $3097.8 \mathrm{ba}$ & $8527 \mathrm{a}$ \\
F33 & $11.1 \mathrm{bc}$ & $49.74 \mathrm{bac}$ & $89.98 \mathrm{ba}$ & $2893.5 \mathrm{ba}$ & $7436 \mathrm{ba}$ \\
F35 & $14.74 \mathrm{bac}$ & $76.59 \mathrm{a}$ & $76.68 \mathrm{ba}$ & $2531.3 \mathrm{bac}$ & $7038 \mathrm{ba}$ \\
F36 & $12.95 \mathrm{bac}$ & $39.64 \mathrm{bac}$ & $108.04 \mathrm{a}$ & $2780 \mathrm{bac}$ & $8088 \mathrm{a}$ \\
F5 & $8.64 \mathrm{bc}$ & $24 \mathrm{bc}$ & $63.85 \mathrm{bac}$ & $2085.4 \mathrm{bac}$ & $6723 \mathrm{bac}$ \\
F7 & $11.05 \mathrm{bc}$ & $34.31 \mathrm{bac}$ & $50.59 \mathrm{bac}$ & $2014 \mathrm{bac}$ & $5581 \mathrm{bc}$ \\
F8 & $15.07 \mathrm{ba}$ & $67.03 \mathrm{ba}$ & $80.42 \mathrm{ba}$ & $2608.8 \mathrm{bac}$ & $7558 \mathrm{ba}$ \\
F9 & $12.95 \mathrm{bac}$ & $54.86 \mathrm{ba}$ & $73.67 \mathrm{ba}$ & $2595.7 \mathrm{bac}$ & $7383 \mathrm{ba}$ \\
\hline Mean & 11.06 & 48.74 & 76.57 & 2490 & 7004.4 \\
CV (\%) & 13.93 & 81.56 & 133.91 & 78.15 & 9.87 \\
p $\leq 0.05$ & 0.0045 & $<0.0001$ & $<0.0001$ & $<0.0001$ & $<0.0001$ \\
\hline \multirow{2}{*}{ family } & 0.4847 & 0.8265 & 0.9683 & 0.9961 & 0.8993 \\
\hline & & & & & \\
\hline
\end{tabular}

\subsection{Growth and Development of Cocoa Trees Region by Region with Significant Region * Family Interactions}

The parameters with significant region * family interactions were mortality rate, collar diameter, plant height and number of living leaves. For these parameters, family rankings were made by region. Mortality rate showed a significant difference between families in Divo and Toumodi and homogeneity in Djekanou and Yamoussoukro (Table 5). The highest mortality rates were obtained by family F19 (39\%) in Divo and families F2 (80.67\%), F7 (75\%), F19 (83\%) and F31 $(78 \%)$ in Toumodi. On the other hand, the lowest mortality rates were presented by families F1 (4.05\%), F5 (4.05\%), F9 (4.05\%), F10 (4.05\%), F21 (2.08\%), F22 (5.04\%), F32 (3.56\%) and F35 (2.08\%) at Divo and family F29 (19.33\%) at Toumodi.

Collar diameter showed a significant difference between families in all regions. The best collar diameters were obtained by family F19 $(2.43 \mathrm{~cm})$ in Divo, F31 $(1.77 \mathrm{~cm})$ in Djekanou, F14 $(2.01 \mathrm{~cm})$ and F16 (2.02) in Toumodi and F31 $(2.15 \mathrm{~cm})$ in Yamoussoukro. On the other hand, the smallest diameters at the collar were presented by families F7 $(1.22 \mathrm{~cm})$ and F17 $(1.33 \mathrm{~cm})$ in Divo, F1 $(1.14 \mathrm{~cm})$ and F28 $(1.04 \mathrm{~cm})$ in Djekanou, F1 $(1.14 \mathrm{~cm})$ and F13 $(1.08 \mathrm{~cm})$ in Toumodi and F1, F2, F3, F5, F7, F8, F9, F10, F11, F13, F14, F16, F17, F20, F21, F22, F25, F27, F32, F35 and F36 with values that varied from 1 to $1.28 \mathrm{~cm}$ in Yamoussoukro (Table 5). 
Table 5. Comparison of families according to mortality rate and crown diameter (parameters with significant region * family interaction) within regions.

\begin{tabular}{|c|c|c|c|c|c|c|c|c|}
\hline \multirow[b]{2}{*}{ Family } & \multicolumn{4}{|c|}{ Mortality rate (\%) } & \multicolumn{4}{|c|}{ Collar diameter $(\mathrm{cm})$} \\
\hline & Divo & Djekanou & Toumodi & Yamoussoukro & Divo & Djekanou & Toumodi & Yamoussoukro \\
\hline $\mathrm{F} 1$ & $4.05 \mathrm{dc}$ & $22.33 \mathrm{a}$ & $61 \mathrm{ba}$ & $73.22 \mathrm{a}$ & $1.74 \mathrm{c} \mathrm{bd}$ & $1.14 \mathrm{ef}$ & $1.14 \mathrm{ed}$ & $1.05 \mathrm{~b}$ \\
\hline F10 & $4.05 \mathrm{dc}$ & $33.67 \mathrm{a}$ & $36 \mathrm{bac}$ & $50.14 \mathrm{a}$ & $1.40 \mathrm{cbd}$ & $1.31 \mathrm{edfc}$ & 1.47 ebdac & $1.08 \mathrm{~b}$ \\
\hline $\mathrm{F} 11$ & $8 \mathrm{bdc}$ & $30.67 \mathrm{a}$ & $69.33 \mathrm{ba}$ & $68.48 \mathrm{a}$ & $1.35 \mathrm{cbd}$ & $1.23 \mathrm{edfc}$ & $1.36 \mathrm{ebdc}$ & $1.04 \mathrm{~b}$ \\
\hline $\mathrm{F} 12$ & $11 \mathrm{bdc}$ & $33.33 \mathrm{a}$ & $36 \mathrm{bac}$ & $72.62 \mathrm{a}$ & $1.42 \mathrm{cbd}$ & $1.23 \mathrm{edfc}$ & $1.77 \mathrm{bdac}$ & $1.35 \mathrm{ba}$ \\
\hline F13 & $7.05 \mathrm{bdc}$ & $9.03 \mathrm{a}$ & $52.67 \mathrm{bac}$ & $99.58 \mathrm{a}$ & $1.68 \mathrm{cbd}$ & $1.16 \mathrm{edf}$ & $1.08 \mathrm{e}$ & $1 \mathrm{~b}$ \\
\hline F14 & $11.69 \mathrm{bdc}$ & $25.0 \mathrm{a}$ & $36.33 \mathrm{bac}$ & $60.14 \mathrm{a}$ & $1.63 \mathrm{cbd}$ & $1.35 \mathrm{edfc}$ & $2.01 \mathrm{a}$ & $1.26 \mathrm{~b}$ \\
\hline F15 & $24.66 \mathrm{bdac}$ & $11.0 \mathrm{a}$ & $44.67 \mathrm{bac}$ & $37.67 \mathrm{a}$ & $1.88 \mathrm{cbd}$ & 1.47 ebdac & 1.55 ebdac & $1.34 \mathrm{ba}$ \\
\hline F16 & $33.33 \mathrm{ba}$ & $25.0 \mathrm{a}$ & $36 \mathrm{bac}$ & $77.48 \mathrm{a}$ & $2.04 \mathrm{~b}$ & $1.40 \mathrm{ebdfc}$ & $2.02 \mathrm{a}$ & $1.22 \mathrm{~b}$ \\
\hline F17 & $11.69 \mathrm{bdc}$ & $36.33 \mathrm{a}$ & $36.33 \mathrm{bac}$ & 85.81 a & $1.33 \mathrm{~cd}$ & $1.34 \mathrm{edfc}$ & 1.65 ebdac & $1 \mathrm{~b}$ \\
\hline F19 & $39 \mathrm{a}$ & $17.0 \mathrm{a}$ & $83 \mathrm{a}$ & 78.77 a & $2.43 \mathrm{a}$ & $1.57 \mathrm{bac}$ & 1.6 ebdac & $1.85 \mathrm{ba}$ \\
\hline $\mathrm{F} 2$ & $30.67 \mathrm{bac}$ & $41.67 \mathrm{a}$ & $80.67 \mathrm{a}$ & $72.62 \mathrm{a}$ & $1.49 \mathrm{cbd}$ & 1.27 edfc & 1.5 ebdac & $1.02 \mathrm{~b}$ \\
\hline $\mathrm{F} 20$ & $11 \mathrm{bdc}$ & $17.36 \mathrm{a}$ & $41.33 \mathrm{bac}$ & $72.62 \mathrm{a}$ & $1.64 \mathrm{cbd}$ & $1.35 \mathrm{edfc}$ & 1.60 ebdac & $1.07 \mathrm{~b}$ \\
\hline $\mathrm{F} 21$ & $2.08 \mathrm{~d}$ & $19.33 \mathrm{a}$ & $47.33 \mathrm{bac}$ & $65.14 \mathrm{a}$ & $1.62 \mathrm{cbd}$ & $1.41 \mathrm{ebdfc}$ & $1.74 \mathrm{bdac}$ & $1.11 \mathrm{~b}$ \\
\hline $\mathrm{F} 22$ & $5.04 \mathrm{dc}$ & $13.54 \mathrm{a}$ & $42 \mathrm{bac}$ & $54.72 \mathrm{a}$ & $1.39 \mathrm{cbd}$ & $1.39 \mathrm{ebdfc}$ & 1.44 ebdac & $1 \mathrm{~b}$ \\
\hline $\mathrm{F} 24$ & $16.67 \mathrm{bdac}$ & $11.0 \mathrm{a}$ & $66.33 \mathrm{bac}$ & 50.67 a & $1.53 \mathrm{cbd}$ & $1.21 \mathrm{edfc}$ & $1.78 \mathrm{bac}$ & $1.45 \mathrm{ba}$ \\
\hline $\mathrm{F} 25$ & $23 \mathrm{bdac}$ & $16.67 \mathrm{a}$ & $58 \mathrm{bac}$ & $72.62 \mathrm{a}$ & $1.65 \mathrm{cbd}$ & $1.20 \mathrm{edfc}$ & 1.38 ebdac & $1.02 \mathrm{~b}$ \\
\hline F26 & $14.69 \mathrm{bdc}$ & $25 \mathrm{a}$ & $55.67 \mathrm{bac}$ & $61.81 \mathrm{a}$ & $1.75 \mathrm{cbd}$ & 1.53 ebdac & 1.75 bdac & $1.86 \mathrm{ba}$ \\
\hline $\mathrm{F} 27$ & $11 \mathrm{bdc}$ & $27.67 \mathrm{a}$ & $36 \mathrm{bac}$ & $66.81 \mathrm{a}$ & $1.61 \mathrm{cbd}$ & 1.27 edfc & $1.81 \mathrm{bac}$ & $1.18 \mathrm{~b}$ \\
\hline F28 & $7.05 \mathrm{bdc}$ & $9.03 \mathrm{a}$ & $61.33 \mathrm{bac}$ & $83.44 \mathrm{a}$ & $1.36 \mathrm{cbd}$ & $1.04 \mathrm{f}$ & 1.59 ebdac & $1.6 \mathrm{ba}$ \\
\hline F29 & $14.69 \mathrm{bdc}$ & $14.69 \mathrm{a}$ & $19.33 \mathrm{c}$ & $34.37 \mathrm{a}$ & $1.55 \mathrm{cbd}$ & $1.29 \mathrm{edfc}$ & $1.75 \mathrm{bdac}$ & $1.51 \mathrm{ba}$ \\
\hline F3 & $6.03 \mathrm{bdc}$ & $25.33 \mathrm{a}$ & $36 \mathrm{bac}$ & $65.14 \mathrm{a}$ & $1.89 \mathrm{cbd}$ & 1.33 edfc & 1.63 ebdac & $1.09 \mathrm{~b}$ \\
\hline $\mathrm{F} 30$ & $6.03 \mathrm{bdc}$ & $13.67 \mathrm{a}$ & $38.67 \mathrm{bac}$ & $74.1 \mathrm{a}$ & $1.77 \mathrm{cbd}$ & 1.478 ebdac & $1.22 \mathrm{ebdc}$ & $1.62 \mathrm{ba}$ \\
\hline $\mathrm{F} 31$ & $11 \mathrm{bdc}$ & $22.33 \mathrm{a}$ & $78 \mathrm{a}$ & $80.48 \mathrm{a}$ & $1.9 \mathrm{cbd}$ & $1.77 \mathrm{a}$ & $1.175 \mathrm{edc}$ & $2.15 \mathrm{a}$ \\
\hline F32 & $3.56 \mathrm{dc}$ & $13.02 \mathrm{a}$ & $50 \mathrm{bac}$ & $41.11 \mathrm{a}$ & $1.74 \mathrm{cbd}$ & $1.31 \mathrm{edfc}$ & $1.51 \mathrm{ebdac}$ & $1.19 \mathrm{~b}$ \\
\hline F33 & $9.72 \mathrm{bdc}$ & $14.0 \mathrm{a}$ & $69.33 \mathrm{ba}$ & $83.44 \mathrm{a}$ & $1.86 \mathrm{cbd}$ & 1.47 ebdac & $1.3 \mathrm{ebdc}$ & $1.37 \mathrm{ba}$ \\
\hline F35 & $2.08 \mathrm{~d}$ & $36.0 \mathrm{a}$ & $52.67 \mathrm{bac}$ & $76.14 \mathrm{a}$ & $1.44 \mathrm{cbd}$ & 1.56 bdac & 1.55 ebdac & $1.26 \mathrm{~b}$ \\
\hline F36 & $7.05 \mathrm{bdc}$ & $16.67 \mathrm{a}$ & $47.33 \mathrm{bac}$ & $63.81 \mathrm{a}$ & $1.96 \mathrm{cb}$ & $1.74 \mathrm{ba}$ & $1.51 \mathrm{ebdac}$ & $1.28 \mathrm{~b}$ \\
\hline F5 & $4.05 \mathrm{dc}$ & $20.36 \mathrm{a}$ & $25.33 \mathrm{bc}$ & $85.29 \mathrm{a}$ & $1.47 \mathrm{cbd}$ & $1.24 \mathrm{edfc}$ & $1.8 \mathrm{ba}$ & $1.2 \mathrm{~b}$ \\
\hline F7 & $33.33 \mathrm{ba}$ & $41.67 \mathrm{a}$ & $75 \mathrm{a}$ & $72.97 \mathrm{a}$ & $1.22 \mathrm{~d}$ & $1.54 \mathrm{ebdac}$ & $1.178 \mathrm{edc}$ & $1.2 \mathrm{~b}$ \\
\hline F8 & $16.33 \mathrm{bdac}$ & $25.0 \mathrm{a}$ & $22.33 \mathrm{bc}$ & $61.85 \mathrm{a}$ & $1.68 \mathrm{cbd}$ & $1.367 \mathrm{edfc}$ & $1.86 \mathrm{ba}$ & $1.2 \mathrm{~b}$ \\
\hline F9 & $4.05 \mathrm{dc}$ & $11.0 \mathrm{a}$ & $47.33 \mathrm{bac}$ & $65.33 \mathrm{a}$ & $1.54 \mathrm{cbd}$ & 1.34 edfc & 1.61 ebdac & $1.03 \mathrm{~b}$ \\
\hline moyenne & 12.79 & 21.87 & 49.89 & 67.77 & 1.64 & 1.36 & 1.61 & 1.28 \\
\hline CV (\%) & 10.99 & 14.71 & 29.67 & 24.18 & 44.71 & 30.23 & 29.66 & 32.56 \\
\hline $\mathrm{p} \leq 0.05$ & $<0.0001$ & 0.1522 & $<0.0001$ & 0.9834 & $<0.0001$ & $<0.0001$ & $<0.0001$ & $<0.0001$ \\
\hline
\end{tabular}

In each column, the means followed by the same letter are not statistically different at the $5 \%$ level. 
Plant height showed a significant difference between families in all regions (Table 6). The greatest plant heights were obtained by families F13 $(118.62 \mathrm{~cm})$, F15 $(124.79 \mathrm{~cm}), F 19(122.92 \mathrm{~cm})$ and F31 $(116.53 \mathrm{~cm})$ in Divo, F31 $(113 \mathrm{~cm})$ and F36 $(109.3 \mathrm{~cm})$ in Djekanou, F8 $(107.29 \mathrm{~cm})$, F14 $(112.78 \mathrm{~cm})$, F16 $(114.57$ $\mathrm{cm})$, F26 $(114.44 \mathrm{~cm})$, F28 $(107.79 \mathrm{~cm})$ and F29 $(111.07 \mathrm{~cm})$ in Toumodi and $\mathrm{F} 19(118 \mathrm{~cm})$ and F31 $(120.5 \mathrm{~cm})$ in Yamoussoukro. On the other hand, the smallest plant heights were shown by families F7 $(81.48 \mathrm{~cm})$, F10 $(87.16 \mathrm{~cm})$, F17 $(86.94 \mathrm{~cm})$ and F35 $(87.17 \mathrm{~cm})$ in Divo, F2 $(71.38 \mathrm{~cm})$ F5 $(72.35 \mathrm{~cm})$, F11 $(73.96 \mathrm{~cm})$, F24 $(70.69 \mathrm{~cm})$ and F25 $(71.07 \mathrm{~cm})$ in Djekanou, F31 $(64.38 \mathrm{~cm})$ in Toumodi and F2 $(36.5 \mathrm{~cm}), \mathrm{F} 13(36.6 \mathrm{~cm})$ and F22 $(39.71 \mathrm{~cm})$ in Yamoussoukro.

The number of live leaves showed a significant difference between families in all regions. The highest numbers of live leaves were obtained by families F19 (62.46 leaves) and F31 (49.81 leaves) in Divo, F19 (38.93 leaves), F31 (50.75 leaves) and F36 (36.76 leaves) in Djekanou, F14 (21.44 leaves) in Toumodi and F19 (67 leaves), F26 (49.63 leaves) and F31 (77 leaves) in Yamoussoukro. On the other hand, the smallest number of living leaves was presented by families F7 (12.67 leaves) in Divo, F1 (13.43 leaves) in Djekanou, F25 (1.8 leaves) and F28 (0.57 leaves) in Toumodi and F1, F2, F3, F5, F7, F9, F10, F11, F12, F13, F14, F15, F16, F17, F20, F21, F22, F25, F27, F28, F32, F35 and F36 with values that varied from 6.33 to 27 live leaves in Yamoussoukro (Table 6).

Table 6. Comparison of families according to height and number of living leaves parameters with significant region * family interaction) within regions.

\begin{tabular}{|c|c|c|c|c|c|c|c|c|}
\hline \multirow[b]{2}{*}{ Family } & \multicolumn{4}{|c|}{ Plant heights $(\mathrm{cm})$} & \multicolumn{4}{|c|}{ Number of living leaves } \\
\hline & Divo & Djekanou & Toumodi & Yamoussoukro & Divo & Djekanou & Toumodi & Yamoussoukro \\
\hline $\mathrm{F} 1$ & 100.05 bdac & $82.46 \mathrm{bac}$ & 83.29 ba & $61.25 \mathrm{ba}$ & $34.17 \mathrm{bcd}$ & $13.43 \mathrm{e}$ & $3.79 \mathrm{bc}$ & $10.5 \mathrm{c}$ \\
\hline F10 & $87.16 \mathrm{dc}$ & $78.85 \mathrm{bac}$ & 89.61 ba & 59.82 ba & $26.11 \mathrm{bcd}$ & $16.21 \mathrm{ed}$ & $10.13 \mathrm{bac}$ & $17.18 \mathrm{c}$ \\
\hline F11 & 97.14 bdac & $73.96 \mathrm{c}$ & 87.55 ba & $65.6 \mathrm{ba}$ & $19.70 \mathrm{~cd}$ & $18.48 \mathrm{ced}$ & $12.636 \mathrm{bac}$ & $13.43 \mathrm{c}$ \\
\hline $\mathrm{F} 12$ & $94.57 \mathrm{bdc}$ & $82 \mathrm{bac}$ & $94.13 \mathrm{ba}$ & $68.08 \mathrm{ba}$ & $20.41 \mathrm{~cd}$ & 21.13 cebd & $7.30 \mathrm{bc}$ & $16.83 \mathrm{c}$ \\
\hline F13 & $118.62 \mathrm{ba}$ & 88.65 bac & 75 ba & $36.6 \mathrm{~b}$ & $38.09 \mathrm{bcd}$ & 15.77 ed & $3.71 \mathrm{bc}$ & $7.51 \mathrm{c}$ \\
\hline F14 & $95.40 \mathrm{bdc}$ & $80.48 \mathrm{bac}$ & $112.78 \mathrm{a}$ & $60.5 \mathrm{ba}$ & $26.81 \mathrm{bcd}$ & $18.11 \mathrm{ced}$ & $21.44 \mathrm{a}$ & $20.22 \mathrm{c}$ \\
\hline F15 & $124.79 \mathrm{a}$ & $101.03 \mathrm{bac}$ & 85.55 ba & 73.46 ba & $40.22 \mathrm{bc}$ & 29.81 cebd & $8.7 \mathrm{bac}$ & $25.33 c$ \\
\hline F16 & $110.98 \mathrm{bac}$ & $86.67 \mathrm{bac}$ & $114.57 \mathrm{a}$ & $74.6 \mathrm{ba}$ & $40.36 \mathrm{bc}$ & $33.19 \mathrm{cbd}$ & $13.48 \mathrm{bac}$ & $23 \mathrm{c}$ \\
\hline F17 & $86.94 \mathrm{dc}$ & $74.65 \mathrm{bc}$ & $101.52 \mathrm{ba}$ & $51.53 \mathrm{ba}$ & $21.47 \mathrm{~cd}$ & 27.57 cebd & $8.57 \mathrm{bac}$ & $11.33 \mathrm{c}$ \\
\hline F19 & $122.92 \mathrm{ba}$ & $97.2 \mathrm{bac}$ & 81.17 ba & $118 \mathrm{a}$ & $62.46 \mathrm{a}$ & $38.93 \mathrm{~b}$ & $6.67 \mathrm{bc}$ & $67 \mathrm{ba}$ \\
\hline $\mathrm{F} 2$ & $94.68 \mathrm{bdc}$ & $71.38 c$ & $92.43 \mathrm{ba}$ & $36.5 \mathrm{~b}$ & $17.88 \mathrm{~cd}$ & $17.10 \mathrm{ed}$ & $9.57 \mathrm{bac}$ & $8.83 c$ \\
\hline $\mathrm{F} 20$ & $104.59 \mathrm{bdac}$ & $89.87 \mathrm{bac}$ & $90.76 \mathrm{ba}$ & $50.83 \mathrm{ba}$ & $23.91 \mathrm{~cd}$ & $20.33 \mathrm{ced}$ & $17.38 \mathrm{ba}$ & $6.33 c$ \\
\hline $\mathrm{F} 21$ & $99.92 \mathrm{bdac}$ & $85.41 \mathrm{bac}$ & $97.11 \mathrm{ba}$ & $61.94 \mathrm{ba}$ & $23.61 \mathrm{~cd}$ & 24.45 cebd & $7.05 b c$ & $12.38 \mathrm{c}$ \\
\hline $\mathrm{F} 22$ & $93.74 \mathrm{bdc}$ & $102.91 \mathrm{bac}$ & $101.43 \mathrm{ba}$ & $39.71 \mathrm{~b}$ & $18.65 \mathrm{~cd}$ & 29.67 cebd & $4.29 \mathrm{bc}$ & $8 c$ \\
\hline $\mathrm{F} 24$ & $101.09 \mathrm{bdac}$ & $70.69 c$ & $104.92 \mathrm{ba}$ & $82.59 \mathrm{ba}$ & $25.5 \mathrm{bcd}$ & $16.56 \mathrm{ed}$ & $10.42 \mathrm{bac}$ & $38.82 \mathrm{bc}$ \\
\hline F25 & 99.49 bdac & $71.07 \mathrm{c}$ & $74.2 \mathrm{ba}$ & $57.75 \mathrm{ba}$ & $23.96 \mathrm{~cd}$ & $16.33 \mathrm{ed}$ & $1.8 \mathrm{c}$ & $7.5 \mathrm{c}$ \\
\hline
\end{tabular}




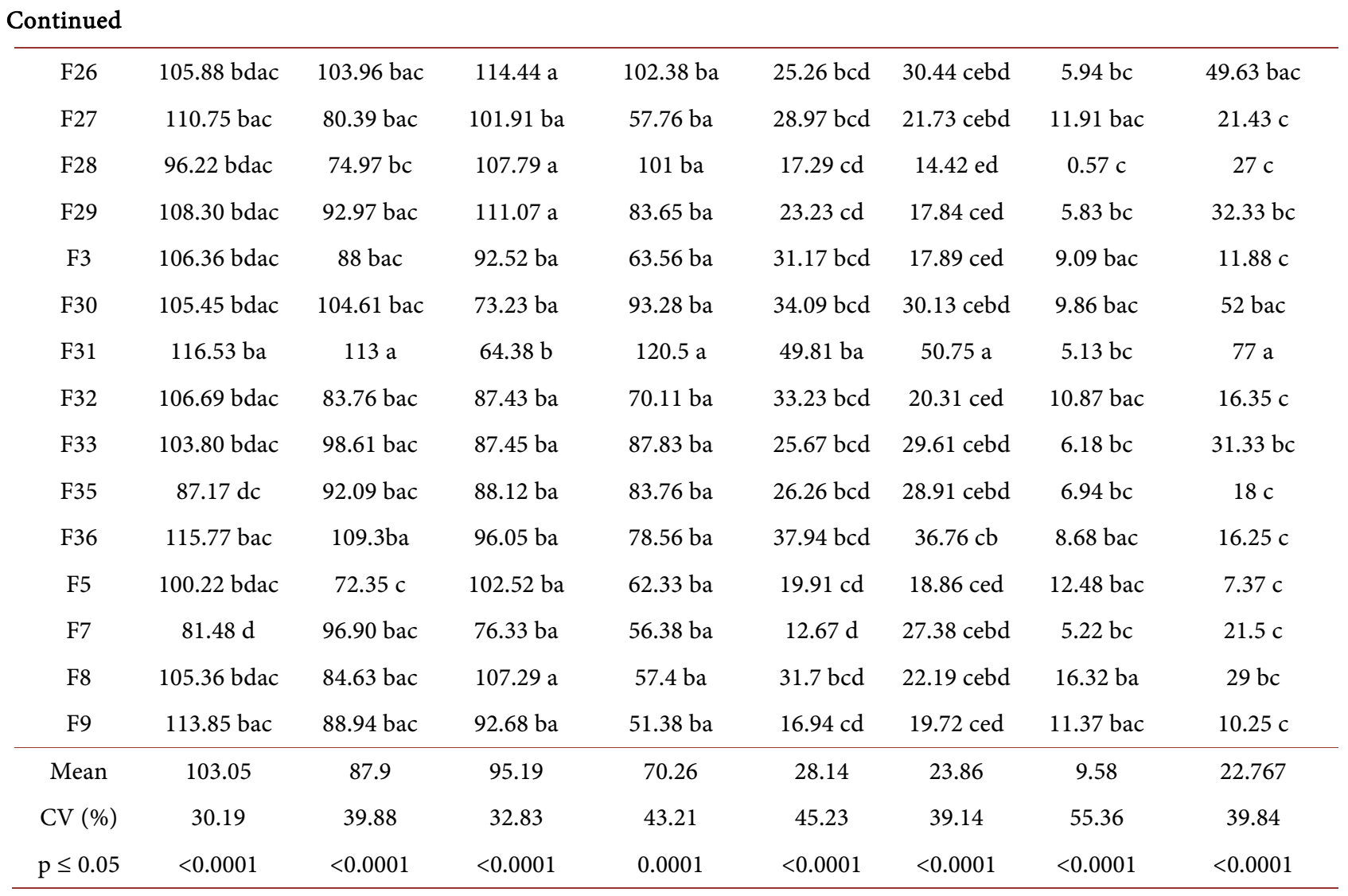

In each column, the means followed by the same letter are not statistically different at the $5 \%$ level.

\subsection{Correlation between Mortality Rate, Development and Growth Parameters and Physiological Parameters}

The Pearson correlation matrix (Table 7) revealed the strongest relationships between mortality rate, development and growth parameters and physiological parameters of young cocoa trees. Thus, development and growth parameters such as crown diameter, plant height, crown height, number of live leaves and leaf area were negatively correlated with mortality rate respectively $(-0.52)$, $(-0.72),(-0.89),(-0.96),(-0.5)$ and $(-0.81)$. Similarly, leaf specific mass, a physiological parameter was negatively correlated with development and growth parameters respectively $(-0.78),(-0.94),(-0.92),(-0.94)$ and $(-0.47)$ except for the number of living leaves. Also, water content, a physiological parameter was negatively correlated with mortality rate $(-0.87)$ and positively correlated with total leaf area (0.94).

\subsection{Projection of Regions According to Development and Physiological Variables of Cocoa Genotypes in Design 1 and 2}

The share of information returned by the two selected axes of the principal component analysis of the regions was $94.27 \%$. Axis 1 expressed $77.67 \%$ of the total variability. The variables well represented on this axis were crown diameter, height, crown ratio, crown height, total leaf area and leaf water content with 
Table 7. Pearson correlation matrix between developmental physiological and mortality variables.

\begin{tabular}{|c|c|c|c|c|c|c|c|c|c|}
\hline variables & diam & haut & Tx cour & hautcou & $n f v$ & $\mathrm{sft}$ & te & $\mathrm{msf}$ & tau mort \\
\hline Diam & 1 & & & & & & & & \\
\hline Haut & 0.77 & 1 & & & & & & & \\
\hline Tx Cour & 0.49 & 0.53 & 1 & & & & & & \\
\hline Hautcou & 0.56 & 0.59 & 0.91 & 1 & & & & & \\
\hline $\mathrm{Nfv}$ & 0.69 & 0.57 & 0.36 & 0.46 & 1 & & & & \\
\hline Sft & 0.49 & 0.4 & 0.21 & 0.28 & 0.53 & 1 & & & \\
\hline $\mathrm{Te}$ & 0.4 & 0.33 & 0.15 & 0.22 & 0.47 & 0.94 & 1 & & \\
\hline $\mathrm{msf}$ & -0.78 & -0.94 & -0.92 & -0.94 & 0.48 & -0.47 & -0.50 & 1 & \\
\hline tau mort & -0.52 & -0.72 & -0.89 & -0.96 & -0.50 & -0.81 & -0.87 & 0.82 & 1 \\
\hline
\end{tabular}

Values in bold are different from 0 with a significance level of $\mathrm{p}<0.000$. Diam $=$ collar diameter; high $=$ plant height; tx yard $=$ crown rate. high yard $=$ crown height. $\mathrm{nfv}=$ number of living leaves. $\mathrm{sft}=$ total leaf area. te $=$ water content. $\mathrm{msf}=$ leaf $\mathrm{specific}$ gravity.

squared cosines ranging from 0.6758 to 0.9230 . This axis was the axis of developmental, growth parameters. Axis 2 expressed $16.58 \%$ of the total variability. The variable that was well represented on this axis was the number of living leaves with a cosine square of 0.6668 . This axis was the axis of a physiological parameter (leaf specific mass) and the mortality rate. The projection of the 4 regions according to the agro-morphological and physiological variables of the cocoa genotypes along axes 1 and 2 is presented in Figure 2. The hierarchical ascending classification of regions gave three classes. Thus the Divo region, which constituted the first class, was home to the progenies with the best development and growth (large diameters, large sizes with more crowns, large crown heights with more functional leaves, with the largest leaf areas, the highest water content), small specific leaf masses and the lowest mortality rate. The regions of Djekanou and Toumodi, which constituted the second class, were home to progenies with intermediate traits. The Yamoussoukro region, which constituted the third class, was home to progenies with poor development and growth except for mortality rate and specific leaf mass.

\subsection{Projection of Progenies as a Function of Developmental and Physiological Variables of Cocoa Genotypes in Planes 1 and 2}

The information returned by the two axes retained from the principal component analysis of the families expressed $64.84 \%$ of the observed variability. Axis 1 expressed $46.95 \%$ of the total variability. The variables collar diameter, plant height, number of living leaves, total leaf area, water content and specific leaf mass constructed this axis of physiological and vigour parameters with squared cosines that varied from 0.5739 to 0.8563 . Axis 2 expressed $17.89 \%$ of the total variability. The variables crown rate with a squared cosine of 0.5123 , crown height with a squared cosine of 0.3702 and mortality rate with a squared cosine 
Biplot (F1 and F2 axis : $94.27 \%$ )

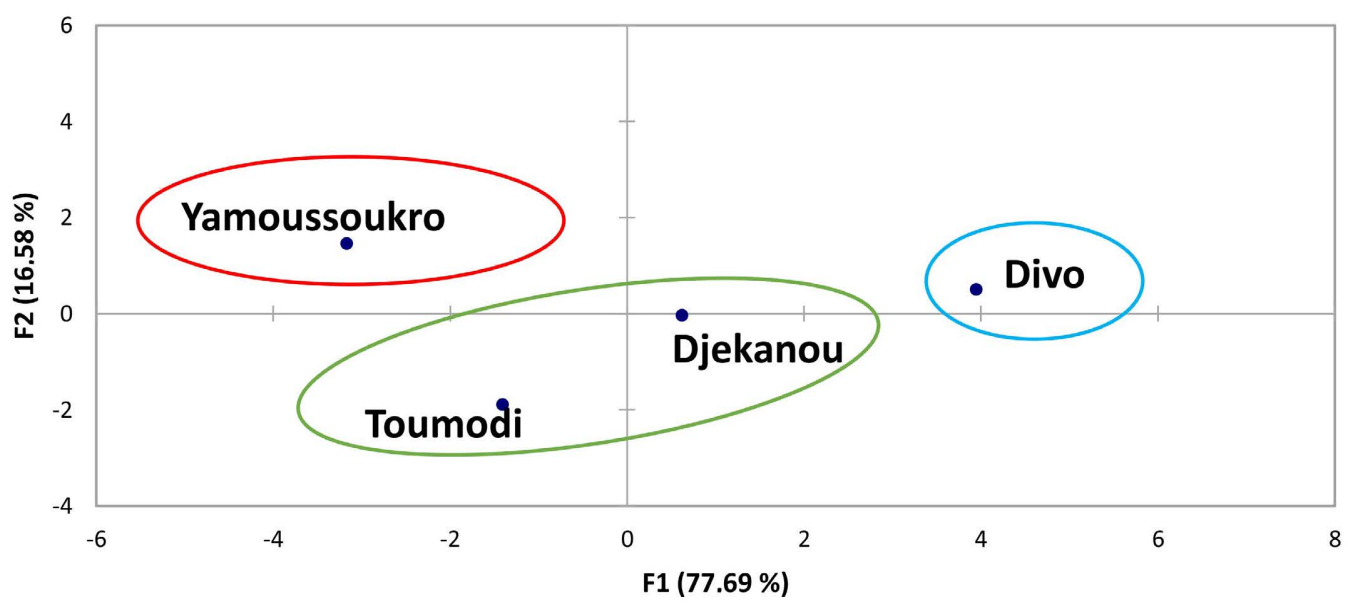

Figure 2. Projection of regions in the $1-2$ plane according to agro-morphological and physiological parameters.

of 0.8070 , constructed this axis of earliness and drought susceptibility (Figure 3 and Figure 4). The results show that within each experimental site after the hierarchical ascending classification, the performance of the families varies significantly between them. Not all families performed equally well and did not rank equally across regions. Indeed, of the 15 best families that were characterised by large crown diameter, large height, large number of living leaves, large leaf area, high water content and low leaf density in Divo (Figure 3(A)), however, of these, only 5 (F30, F15, F19, F31, and F33) were also found to be among the best performers at Djekanou (Figure 3(B)) and Yamoussoukro (Figure 4(A)). None of them were found among the best performers in Toumodi. On the other hand, three other families (F16, F36 and F29) that also performed well in Divo were found in Toumodi (Figure 4(A)) and another (F24) was found to perform well in Yamoussoukro. In the end, the majority of families performing in Toumodi were not found performing in Divo, Djékanou and Yamoussoukro.

\section{Discussion}

The 31 progenies showed highly variable behaviour depending on the evaluation parameters considered.

Thus, the results obtained showed a depressive effect of drought on cocoa varieties. This depressive effect was observed in regions with deficient rainfall such as Toumodi and Yamoussoukro, unlike the control region in a normal rainfall zone (Divo). In this study, progenies showed a decrease in crown diameter and plant height in dry areas. Our results are in line with those of [9] [10] [11] and [7], who showed that vegetative growth (diameter and height) discriminated between water-stressed and drought-stressed varieties. In our study, progenies also showed a reduction in leaf density as well as leaf area in rainfall-deficient areas. [12] stated that one of the first reactions of plants to water deficit is to reduce 


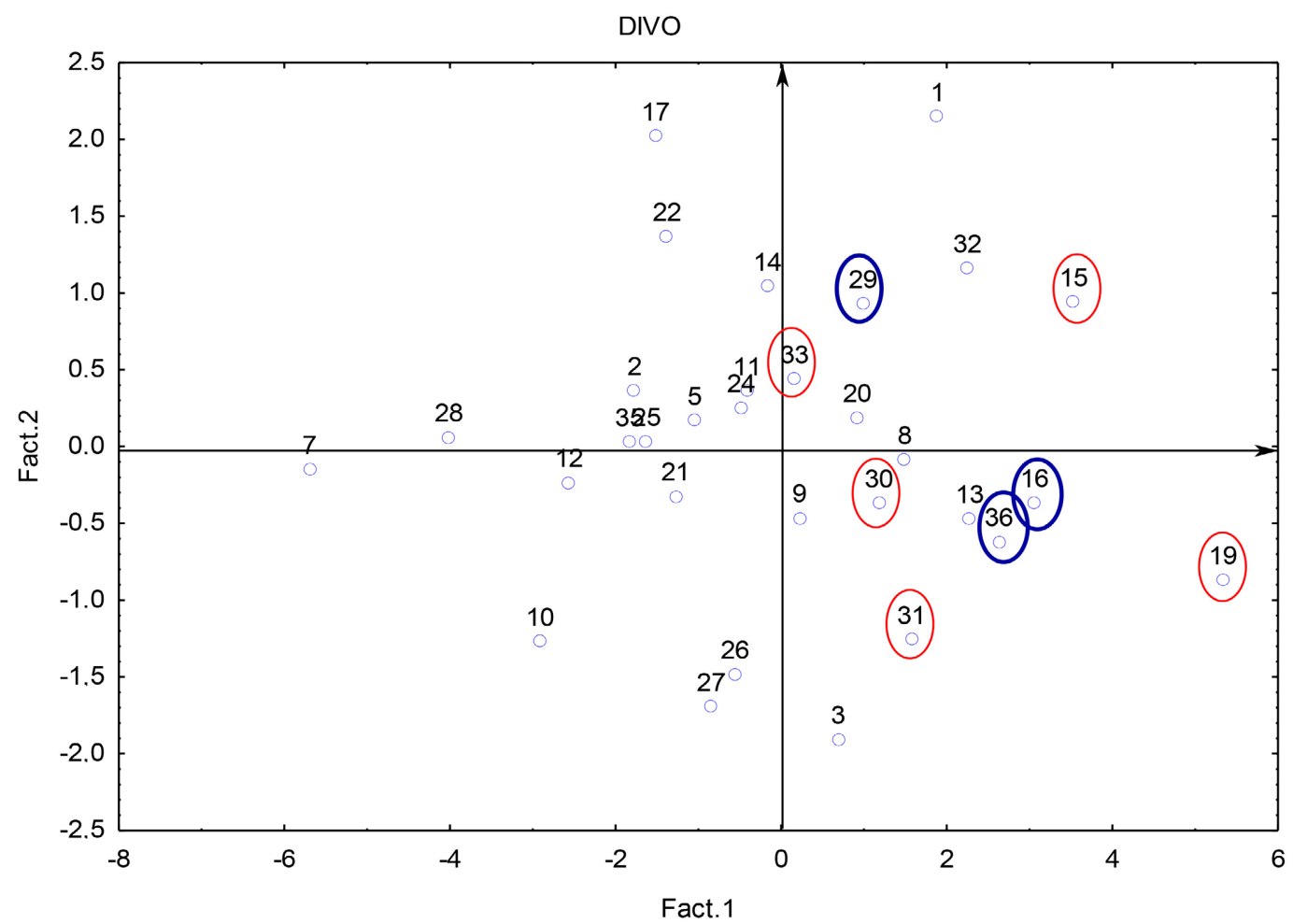

(A)

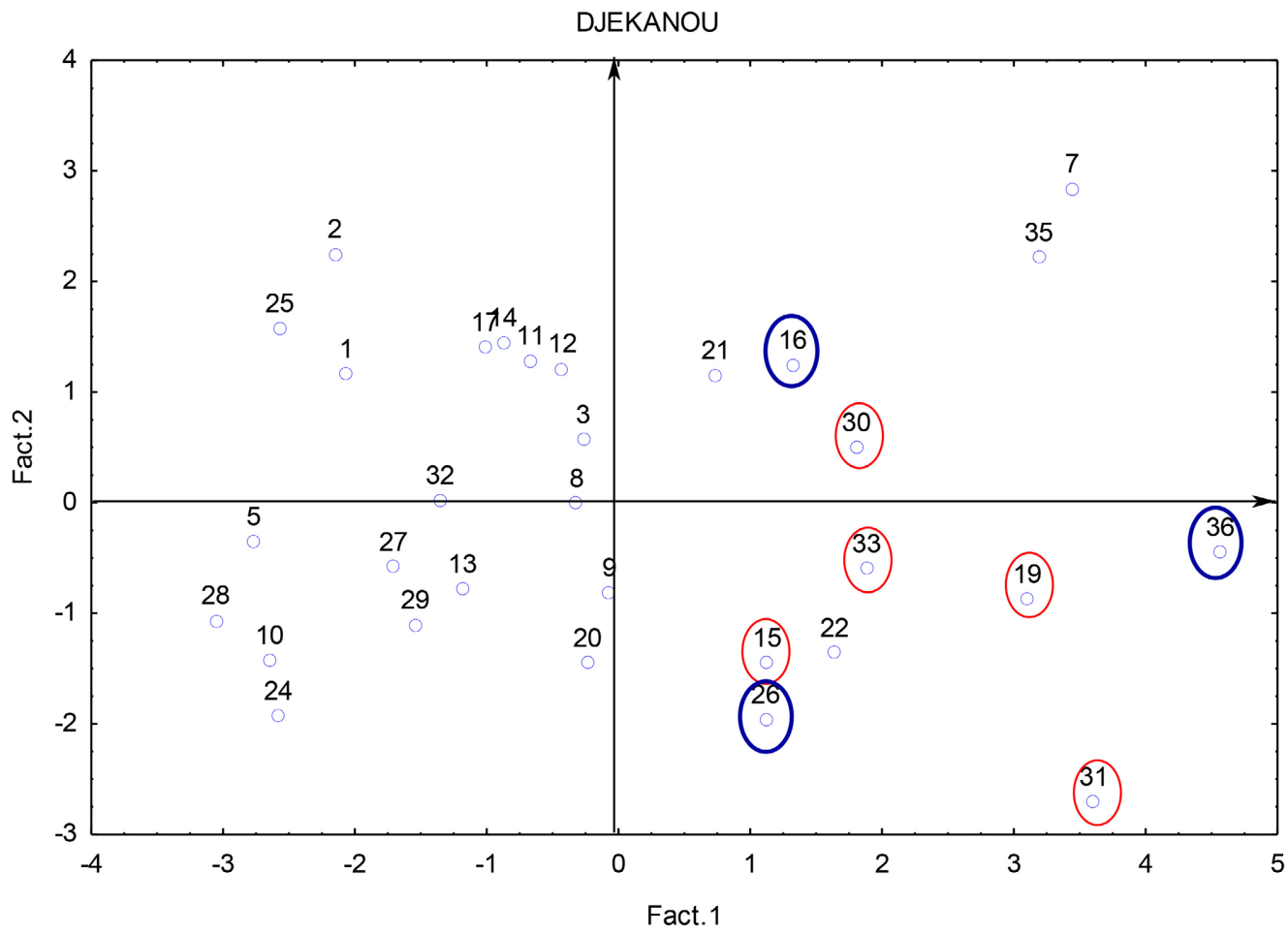

(B)

Figure 3. projection of the progenies of the Divo and Djekanou regions on the principal component analysis planes 1 - 2. (A) projection of Divo progenies in the 1 - 2 plane (The different numbers correspond to the codes of the progenies); (B) Projection of Djekanou progenies in the $1-2$ plane (The different numbers correspond to the codes of the progenies). 
TOUMODI

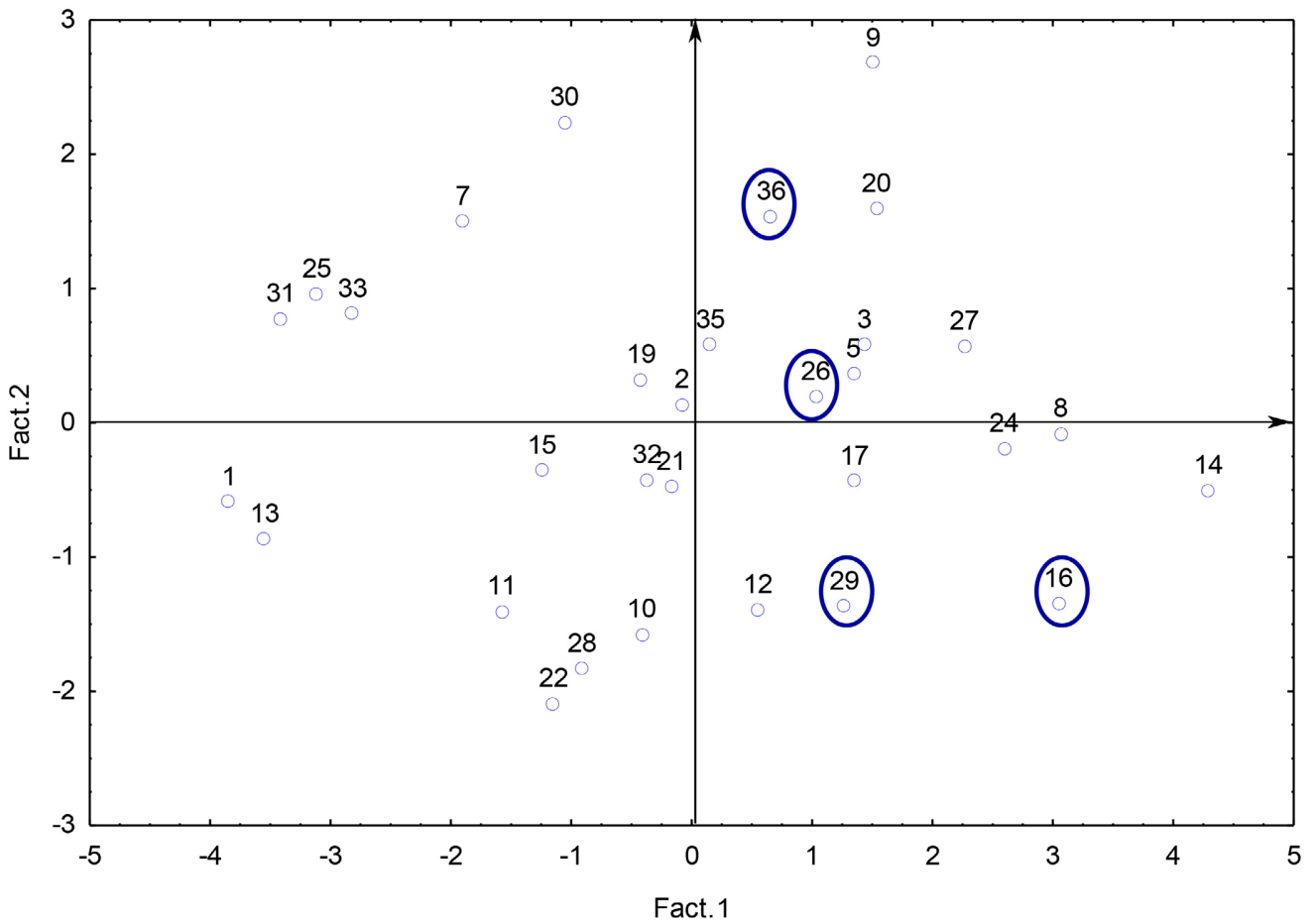

(A)

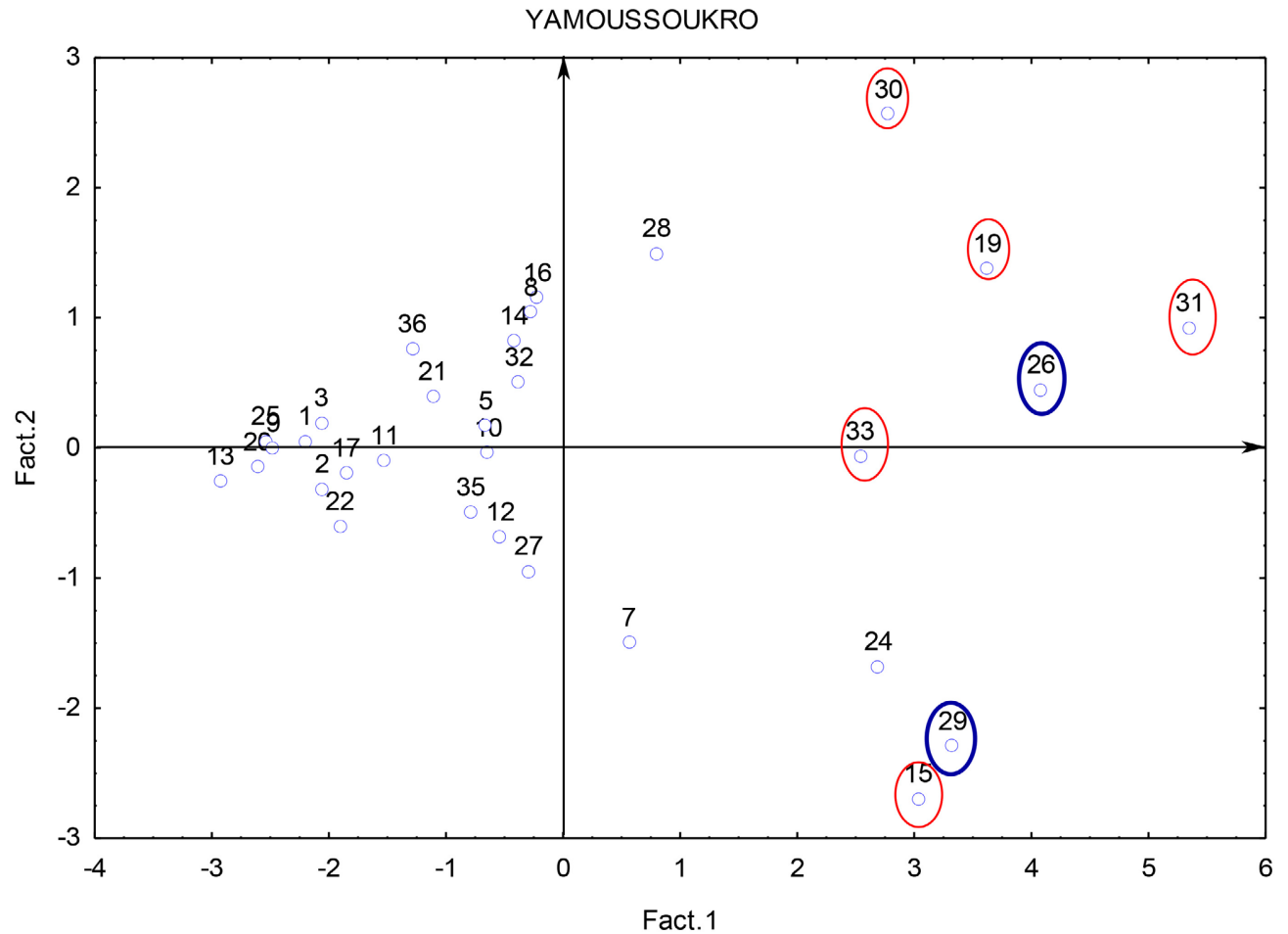

(B)

Figure 4. Projection of the progenies of the Toumodi and Yamoussoukro regions on the principal component analysis planes 1 - 2. (A) projection of Toumodi progenies in the 1 - 2 plane (The different numbers correspond to the codes of the progenies); (B) Projection of Yamoussoukro progenies in the 1 - 2 plane (The different numbers correspond to the codes of the progenies). 
leaf area and the number of functional leaves. Our results also confirm those of [13], who demonstrated that vegetative development under conditions of limited water supply is strongly disturbed, mainly by a significant decrease in the number of functional leaves and leaf area. This decrease in vegetative development is one of the responses of plants to dehydration, in order to conserve water resources and allow the survival of the plant [12]. The effect of water stress due to drought can result, depending on the adaptive strategy of each progeny or variety, in morphological modifications. These modifications affect primary growth [7] [14] [15], thus reducing turgidity and consequently the expansive power of the leaves and favouring the proper development of the underground system [16]. According to [17], leaves of water-stressed plants usually reach smaller final sizes than those of the control.

Water stress due to drought is a determining factor for plant growth in semi-arid regions. It is expressed by a progressive slowing down of primary growth [7] [15], marked by the reduction of leaf cell turgidity. Our study revealed a reduction in leaf water content of progenies in rainfall-deficient areas in Toumodi and Yamoussoukro, in contrast to Divo, a normal rainfall area. Our results corroborate those of [18], who showed that water shortage due to drought induced a decrease in relative water content in stressed plants. [19], noted the same finding and suggested the use of water content as an indicator of the water status of the plant under stress. The water content of cocoa leaves seems to decrease proportionally with the reduction of water content in the soil [20]. [15] noted that this decrease in water content is more rapid in susceptible varieties than in resistant varieties. On the other hand, a high water content under stress conditions has been observed in soybean [21]. On the other hand, [22] showed that genotypes that maintain a high water content in the presence of water stress are tolerant genotypes. Thus, the best progenies from the Yamoussoukro region and the Toumodi region (drier areas) with the highest water content values would appear to be potentially drought tolerant.

The specific leaf mass is one of the important markers in the response of plants to water stress. It can be considered as a simple criterion for selecting genotypes with high water use efficiency under water deficit conditions [23]. The specific leaf mass of progenies in rainfall-deficient areas was higher than in normal rainfall areas. Our results are in line with those of [23] who showed the increase in leaf density in some plants under stress. According to the same author, the increase in specific leaf mass in plants under stress was highly correlated with the reduction in leaf area. Indeed, in this study, the negative correlation $(r=-0.47)$ between specific leaf mass and leaf area confirms this assertion. Indeed, the process of reducing leaf area and increasing leaf specific mass allows plants to "confront" the lack of water by reducing transpiration [23].

\section{Conclusion}

The study of the response to drought in the 31 cocoa progenies tested revealed 
the existence of a large variability for most of the parameters measured. The effect of drought was well marked between regions with deficient rainfall such as Toumodi and Yamoussoukro and those with normal rainfall such as Divo and Djekanou, which seems to be a transition region. Thus, mortality rate and specific leaf mass increased, crown diameter, plant height, leaf area and leaf water content decreased in cocoa trees tested in rainfall-deficient areas. Highly significant positive and negative correlations between agro-morphological and physiological variables were found. The F1, F11, F14, F16, F19, F21, F26, F30, F31, F32, F33 and F36 progenies behaved well in all 4 regions. The significance of the region * family interaction $(\mathrm{p}=0.001)$ showed that the progeny behaved differently from one area to another, depending on the degree of drought severity. Thus, among the fifteen (15) best progenies in Divo (F1, F13, F15, F16, F19, F20, F29, F30, F31, F32, F33, F36, F3, F8 and F9), only five (F30, F15, F19, F31, and F33) repeated the same performance in Djekanou and Yamoussoukro. In addition, 3 other families (F16, F36 and F29) that also performed well in Divo were found in Toumodi. These progenies seemed to adapt well to the drought and were better respectively in Divo (normal rainfall zone) and Djekanou, Yamoussoukro and Toumodi (deficit rainfall zone).

\section{Conflicts of Interest}

The authors declare no conflicts of interest regarding the publication of this paper.

\section{References}

[1] Jérôme, A.N., Arsène, D. and Guillaume, N'g.K. (2014) Crise de l'économie de plantation et modification du paysage agraire dans l'ancienne boucle du cacao: l'exemple de daoukro. European Scientific Journal, 10, 25 p.

[2] Tahi, G.M., Lachenaud, P., N'Guessan, K.F., N'Goran, N.K.J., Pokou, D., Kébé, I.B., Paulin, D., Cilas, C. and Eskes, A.B. (2011) Selection of New Varieties On-Station and On-Farm in Côte d'Ivoire. In: Albertus, E., Ed., Collaborative and Participatory Approaches to Cocoa Variety Improvement. Final Report of the CFC/ICCO/Bioversity International Project on "Cocoa Productivity and Quality Improvement: A Participatory Approach” (2004-2010), CFC, Amsterdam, 42-58. https://agritrop.cirad.fr/560755/

[3] Koua, S.H., Coulibaly, N.A.M.D. and Alloué-Boraud, W.A.M. (2018) Caractérisation des vergers et des maladies de cacao de Côte d'Ivoire: Cas des départements d'Abengourou. Divo et Soubré, 35, 5706-5714. http://www.m.elewa.org/JAPS

[4] FAO (Food and Agriculture Organization of the United Nations) (2017) Données forestières de base pour la REDD+ en Côte d'Ivoire. Cartographie de la dynamique forestière de 1986 à 2015. Publié par l'Organisation des Nations Unies pour l'alimentation et l'agriculture et le Secrétariat Exécutif Permanent REED+, Abidjan, 32 p. http://www.fao.org/publications.

[5] Mathias, T.G., Philippe, L., N’Goran Jeanne, A.K., Kébé Ismael, S., Paulin, D., N'Guessan, K.F., Cilas, C. and Eskes, A. (2010) Second cycle de sélection récurrente du cacaoyer (Theobroma cacao L.) en Côte d'Ivoire: Bilan à mi-parcours et propositions de sorties variétales. 16th International Cocoa Research Conference. Proceed- 
ings: Towards Rational Cocoa Production and Efficient Use of a Sustainable World Cocoa Economy, Bali, 16-21 November 2009, 3-12.

http://publications.cirad.fr

[6] Mathias, T.G., Lachenaud, P., N’Goran Jeanne, A.K. and Eskes, A. (2012) Comportement et utilité des géniteurs Guyanais dans le programme d'amélioration génétique du cacaoyer en Côte d'Ivoire. 17 th Conférence Internationale sur la Recherche Cacaoyère, Yaounde, 15-20 Octobre 2012, 11 p.

http://publications.cirad.fr.

[7] M’bo, K.A.A. (2015) Adaptation des cacaoyers [theobroma cacao l. (malvaceae)] a la sécheresse: étude des paramètres morphologiques, physiologiques, biochimiques et des marqueurs moléculaires de la tolérance. Thèse de doctorat, Université Félix Houphouët-Boigny, Abidjan, 239 p.

[8] Zeghida, A., Amrani, R., Djennadi, F., Ameroun, R., Khldoun, A.A. and Belloucif, M. (2004) Etude de la variabilité de réponse des plantules de blé dur (Triticum durum Desf) à la salinité. Céréaliculture. ITGC, 42. Constantine, 5 p.

[9] Boyer, J. (1971) Etude des principales composantes du microclimat d'une cacaoyère au Cameroun. Importance écologique des variations spatiales et saisonnières. Café-Cacao-Thé, 15, 275-300.

[10] Boyer, J. (1973) Etude particulière des facteurs hydriques de la croissance des cacaoyers. Café Cacao Thé, 17, 189-204.

[11] Alvim, P.T. and Alvim, R. (1978) Relation of Climate to Growth Periodicity in Tropical Trees. In: Tomlinson, P.B. and Zimmermann, M.H., Eds., Tropical Trees as Living Systems, Cambridge University Press, Cambridge, 445-464.

[12] Lebon, E., Pellegrino, A., Tardieu, F. and Lecoeur, J. (2004) Shoot Development in Grapevine Is Affected by the Modular Branching Pattern of the Stem and Intra and Inter-Shoot Trophic Competition. Annals of Botany, 93, 263-274. https://doi.org/10.1093/aob/mch038

[13] Ferryra, R., Sellés, G., Ruiz, R.S. and Sellés, I.M. (2004) Effect of Water Stress Induced at Different Growth Stages on Grapevine cv. Chardonnay on Production and Wine Quality. Acta Horticulturae, 664, 233-236. https://doi.org/10.17660/ActaHortic.2004.664.27

[14] Monneuveux, P. and Memmar, M. (1986) Contribution à l'étude de la résistance à la sécheresse chez le Blé tendre (Triticum aestivum L.) et chez le Blé dur (Triticum durum desf.): Étude de l'accumulation de proline au cours du cycle de développement. Agronomie, 6, 583-590. https://doi.org/10.1051/agro:19860611

https://hal.archives-ouvertes.fr/hal-00884913

[15] Adra, M. (2010) Caractères physiologiques et biochimiques de tolérance du blé dur (Triticum durum Desf.) au stress hydrique. Thèse de doctorat, University of Constantine 1, Constantine, $118 \mathrm{p}$.

[16] Slama, A., Ben Salem, M., Ben Naceur, M. and Zid, E.D. (2005) Les céréales en Tunisie: Production effet de la sécheresse et mécanismes de résistance. Institut national de la recherche agronomique de Tunisie (Inrat). Université de Tunis El Manar, Tunis.

http://www.john-libbeyeurotext.fr/fr/revues/agro biotech/sec/e-docs/00/04/11/2E/t elecharger.md

[17] Granier, C., Inzé, D. and Tardieu, F. (2000) Spatial Distribution Cell Division Rate Can Be Deduced from that of P34 ${ }^{\text {cdc2 }}$ Kinase Activity in Maize Leaves Grown in Contrasting Conditions of Temperature and Water Status. Plant Physiology, 124, 1393-1402. https://doi.org/10.1104/pp.124.3.1393 
[18] Albouchi, A., Sebei, H., Mezni, M.Y. and EL Aouni, M.H. (2000) Influence de la durée d'une alimentation hydrique déficiente sur la production de biomasse, la surface transpirante et la densité stomatique $d$ Acacia cyanophylla. Annales de I INRGREF, 4, 138-161.

[19] Clarck, J.M. and Mac-Caig, T.N. (1982) Excised Leaf Water Relation Capability as an Indicator of Drought Resistance of Triticum Genotypes. Canadian Journal of Plant Science, 62, 571-576. https://doi.org/10.4141/cjps82-086

[20] Bajji, M., Lutts, S. and Kinet, J.-M. (2001) Water Deficit Effects on Solute Contribution to Osmotic Adjustment as a Function of Leaf Ageing in Three Durum Wheat (Triticum durum Desf.) Cultivars Performing Differently in Arid Conditions. Plant Science, 160, 669-681. https://doi.org/10.1016/S0168-9452(00)00443-X

[21] De Raïssac, M. (1984) Réponse physiologique du soja à la sécheresse; étude de la variabilité génétique. Thèse de docteur-ingénieur: Sciences Agronomiques: Institut national polytechnique de Toulouse, ENSAT, Toulouse, $115 \mathrm{p}$.

[22] Nouri, L. (2002) Ajustement osmotique et maintien de l'activité photosynthétique chez le blé dur (Triticum durum. Desf), en condition de déficit hydrique. Thèse de Magistère en Biologie végétale Univ Mentouri, Constantine, 77 p.

[23] Ykhlef, N. (2001) Photosynthèse, activité photochimique et tolérance au déficit hydrique chez le blé dur (Triticum durum Desf). Thèse de doctorat, University of Constantine 1, Constantine. 